\title{
A Review Paper on Knowledge Map Construction for Monitoring the Health of the Company
}

\author{
Arti Gupta, Prof. N.T. Deotale \\ (Computer Science Department, Rastrasant Tukdoji Maharaj Nagpur University, Priyadarshini Bhagwati \\ College Of Engineering, Harpur Nagar, Nagpur, India) \\ (Computer Science Department , Rastrasant Tukdoji Maharaj Nagpur University, Priyadarshini Bhagwati \\ College Of Engineering, Harpur Nagar, Nagpur, India)
}

Abstract: This paper produces the business structure map to understand business performance by analysing the various aspects of the company The business structure map or knowledge map are used to increase the growth of the company by giving some useful measures according to the business criteria. These paper also deals with the different company strategy to mitigate the risk factors. Knowledge Map is helpful for developing such knowledge successfully.

Keywords: business criteria,,business structure, knowledge map, process map ,strategy

\section{I.Introduction}

In earlier days cognitive maps are used that consists nodes and links..In 1976, Robert Axelrod invents cognitive map.[1].Each link represent relationship between the two nodes. The relationship is denoted by the + and the - sign. The + sign indicate the positive relationship and the - sign indicate the negative relationship.

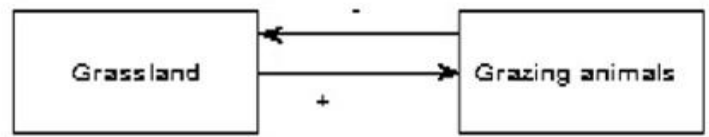

FIG.1 A Cognitive map example

In the above example the grassland and grazing animals are two nodes. The bidirectional arrow indicates its relationship.

As it suggests the quantity of grassland is proportional to quantity of grazing animals and vice versa as grassland increases number of animals is also increased and if grassland decreases number of animals is also decreased. The next extension of cognitive map is fuzzy cognitive map. It also consist nodes and links without restrictions on the nodes limitations. But it also consist weights on each link, arcs or edges. In the Fig no 2 . $\mathrm{C} 1, \mathrm{C} 2, \mathrm{C} 3$ are different nodes or concepts. E12,E31 are the weighted edges from node 1 to 2 and node 3 to 1.the links are unidirectional or bidirectional. Each associated weight has three different causality.

$\mathrm{W} \mathrm{i}, \mathrm{j}=0$, represent no causality

$\mathrm{W} i, j>0$, represent positive causality

$\mathrm{W} i, \mathrm{j}<0$ represent negative causality

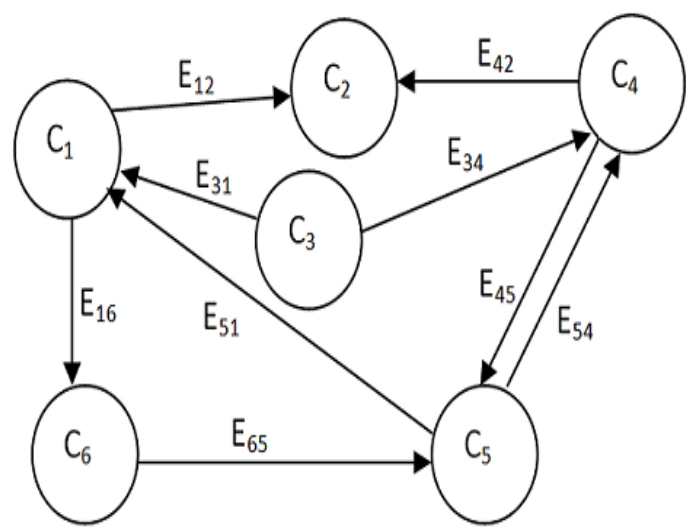

FIG. 2 A Simple FCM example 
An another extension of Fuzzy cognitive Map is Knowledge Map .It consists nodes, arcs and connectors. The only difference between these Fuzzy cognitive Map and Knowledge Map is the use of connectors..The Fuzzy cognitive Map is widely used in various applications like software development project,fault finding system, business purpose and for medical diagnosis. Several advantages of Knowledge Map are,

- $\quad$ It is faster.

- $\quad$ Easy to understand.

- Not require human knowledge.

- Automatically generated.

Applications of Fuzzy cognitive Map cover a wide range of research and industrial areas, such as electrical engineering, medicine, political science, international relations, military science, history, supervisory systems, etc. Examples of specific applications include diagnosis of diseases, analysis of electrical circuits analysis of failure modes effects, fault management in distributed network environment, modelling and analysis of business performance indicators, modelling of supervisory systems, modelling of software development project ,modelling of plant control ,modelling of political affairs in South Africa modelling of virtual worlds , and protein sequence analysis[2].

\subsection{Example Application Areas}

Some example application areas were selected to present the way FCMs were applied and depicted in what follows:

\section{- Medicine}

At the last decade, FCMs have found important applicability in medical diagnosis and decision support In medical domain and in particular for medical decision support tasks, FCM based decision methodologies include an integrated structure for treatment planning management in radiotherapy, a model for specific language impairment, models for bladder and brain tumour characterization , an approach for the pneumonia severity assessment, and a model for the management of urinary tract infections. Stylios et al. proposed FCM architectures for decision support in medicine .

\section{- Engineering}

In this domain, FCMs found a large number of applications, especially in control and prediction. Particularly, FCMs have been used to model and support a plant control system, to construct a system for failure modes and effect analysis, to fine tune fuzzy logic controllers, to model the supervisor of a control system etc.

\section{- Business}

In business, FCMs have found a great applicability. They can be used for product planning, for analysis and decision support. Some interested applications and worthy to be referred are illustrated. Jetter et al. (2006) used the concept of fuzzy front end for ideation, concept development and concept evaluation of new product development. This concept helped various problems managers who faced difficulty in early product development, as well as to systematic approaches to deal with them.

\section{- Production Systems}

FCM can provide an interesting solution to the issue of assessing the factors which are considered to affect the operator's reliability and can be investigated for human reliability in production systems. Bertolini \& Bevilacqua (2010) investigated the human reliability in production systems which act as an excellent means to study a production process and obtain useful indications on the consequences which can be determined by the variation of one or more variables in the system examined.

\section{- Information Technology (IT)}

In information technology (IT) project management, a FCM-based methodology helps to success modelling. Current methodologies and tools used for identifying, classifying and evaluating the indicators of success in IT projects have several limitations

\section{- Environment and Agriculture}

FCMs were applied in ecology and environmental management for: modelling a generic shallow lake ecosystem by augmenting the individual cognitive maps, assessing local knowledge use in agro-forestry management , modelling of interactions among sustainability components of an agro-ecosystem using local knowledge , predicting modelling a New Zealand dry land ecosystem to anticipate pest management outcomes , semiquantitative scenario, with an example from Brazil. 


\section{II.Problem Defination}

In Fuzzy Cognitive Maps number of nodes increases number of arcs also increased. So it becomes complicate to understand. And the process is time consuming. To solve this problem Allen and Marczyk introduced the Knowledge Map concept which was derived from Fuzzy Cognitive Map .The Knowledge Map is very much useful in extracting knowledge and also shows the flow of relationship between various datasets.

The main drawback of Fuzzy Cognitive Map was when too many parameters came into map it become very complex to judge. So KM replaced this drawback by replacing this complex view by segmented view. The segmented view is selected because it reduces redundancy. The segmented theory is based on Design Structure Matrix (DSM) theory. This process reduces complex data to a Knowledge Map .and how to generate Knowledge Maps from complex data sets and how Knowledge Maps can be used to help make better decisions.

\section{III.Related Work}

A number of algorithms for learning FCM model structure have been recently proposed. In general two main learning paradigms are used. Hebbian learning and genetic algorithms. Dickerson and Kosko proposed simple Differential Hebbian Learning law (DHL) to be applied to learning FCMs.[3] The learning process iteratively updates values of weights of all edges from the FCM graph until the desired structure is found.

In 2002 Vazquez presented an extension to DHL algorithm by introducing new rules to update edge values . This new algorithm was called Balanced Differential Algorithm (BDA). The new algorithm eliminates the limitation of DHL method [5] where weight update for an edge connecting two concepts (nodes) is dependent only on the values of these two concepts. However, proposed learning method was applied only to Fuzzy Cognitive Map with binary concept values which significantly restricts its application areas.

Another method based on Hebbian learning was proposed in 2003. Papageorgiou et al.[6] developed an algorithm called Nonlinear Hebbian Learning (NHL) to learn structure of FCM. The main idea behind this method is to update weights associated only with edges that are initially suggested by expert non-zero weights .As a result, the NHL algorithm allows obtaining model that retains structure which is enforced by the expert but at the same it requires human intervention before the learning process starts.

Active Hebbian Algorithm (AHL) introduced by Papageorgiu et al. in 2004 is the next attempt to help in Fuzzy Cognitive .Map development.. [7]This approach introduces and exploits the task of determination of the sequence of activation concepts. The main disadvantage of this approach is that it still requires human intervention.

In 2001 Koulouriotis et al. applied the Genetic Strategy (GS) to learn FCM model structure, weights of relationships, from data. In this method the learning process is based on a collection of input/output pairs which are called examples Its main drawback is the need for multiple state vector sequences which might be difficult to obtain for many real life problems.

Parsopoulos et al. in 2003 applied Particle Swarm Optimization (PSO) [9]method which belongs to the class of Swarm Intelligence algorithms to learn Fuzzy Cognitive Map structure based on a historical data consisting of a sequence of state vectors that leads to a desired fixed-point attractor state. PSO is a population based algorithm which goal is to perform a search by maintaining and transforming a population of individuals. This method improves the quality of resulting FCM model by minimizing an objective function.

Another state of the art learning method for FCM introduced by Stach et al. in 2005 applies real-coded genetic algorithm (RCG.A) to develop FCM model from a set of historical data This approach is very flexible in terms of input data. It can use either one time series or multiple sets of concepts values over successive iterations.

\section{IV.Literature Survey}

Author B. Kosko developed a fuzzy causal algebra for governing causal propagation on FCM.. FCM matrix representation and matrix operations are presented in this context. Dickerson and Kosko proposed a simple Differential Hebbian Learning (DHL) algorithm which iteratively updated the values of the weights until they converged to certain predefined state. The generated FCM then become very complex and unreadable.

J. Aguilar proposed the automated construction of FCM using learning procedure is a new field. These approaches offer the advantage of quantified results but suffer from several drawbacks. First developing the model typically requires a great deal of effort and specialized knowledge outside the domain of interest. Secondly systems involving significant feedback propagates casual influences in complicated chains may be nonlinear in which case a quantitative model may not be possible. Finally numerical data may be hard to come.

J. L. Salmeron proposes to build an Augmented Fuzzy Cognitive Map abased for modelling Critical Success Factors in Learning Management Systems. The study of Critical Success Factors helps decision makers to extract from the multidimensional learning process the core activities that are essential for success. [10] 


\section{Module 1 : Requirement gathering}

\section{V.Proposed Work}

Collect different dataset from various companies and need to create the database to mine knowledge maps from it. These databases can be from financial companies or any companies having major finance background and need to collect revenue, income tax returns and maintenance cost like parameter The database used is financial related, the charted account firm or Market share data of any financial firm.[12] If any dataset is available for research we will use that or we need to create our own. Knowledge Map always represent the concepts. Concept can be anything related to your domain like profit, loss, expenses, customer satisfaction, hr satisfaction etc. So we are keeping the data related to these section.

\section{Module 2 : Knowledge Map Creation}

This is very important step of research as we are creating knowledge maps based on the historical data collected in module1. Knowledge map creation step has 4 steps

- Scatter plot generation

- Fuzzy Rule Generation

- Map construction

- Hubs \& Inactive node identification

\subsection{Scatter plot generation:}

A scatter diagram is a tool for analyzing relationships between two variables. One variable is plotted on the horizontal axis and the other is plotted on the vertical axis. The pattern of the intersecting points can graphically show relationship patterns. Most often a scatter diagram is used to prove or disprove cause and effect relationships. While the diagram shows relationships it does not by itself prove that one variable causes the other. In addition to showing possible cause and effect relationships a scatter diagram can show that two variables are from a common cause that is unknown or that one variable can be used as a surrogate for the other. Scatter diagrams will generally show one of six possible correlations between the variables[12]

- Strong Positive Correlation

The value of $\mathrm{Y}$ clearly increases as the value of $\mathrm{X}$ increases.

- Strong Negative Correlation

The value of $\mathrm{Y}$ clearly decreases as the value of $\mathrm{X}$ increases.

- Weak Positive Correlation

The value of $\mathrm{Y}$ increases slightly as the value of $\mathrm{X}$ increases.

- Weak Negative Correlation

The value of $\mathrm{Y}$ decreases slightly as the value of $\mathrm{X}$ increases.

- Complex Correlation

The value of $\mathrm{Y}$ seems to be related to the value of $\mathrm{X}$, but the relationship is not easily determined.

- No Correlation

There is no demonstrated connection between the two variables.

\subsubsection{When to use it}

- Use a scatter diagram to examine theories about cause-and-effect relationships and to search for root causes of an identified problem.

- Use a scatter diagram to design a control system to ensure that gains from quality improvement efforts are maintained. 
Strong Negative Correlation

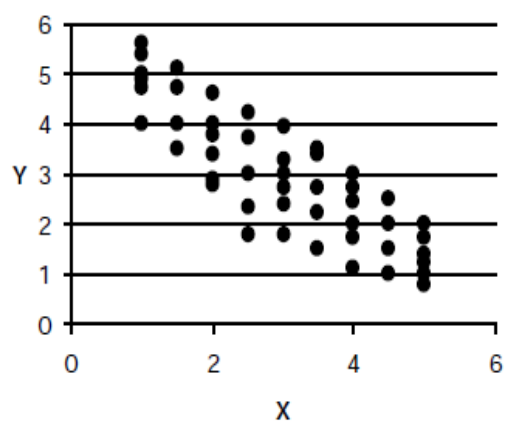

FIG. 3 Scatter plot generation with negative Correlation
Strong Positive Correlation

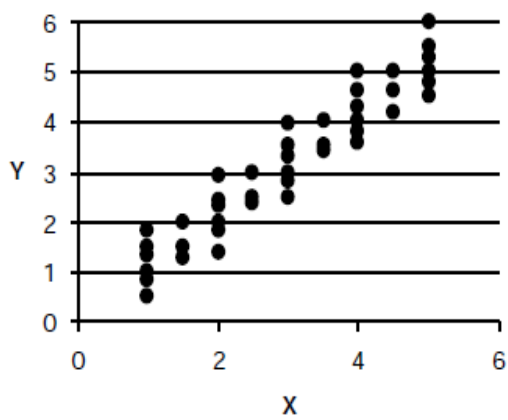

FIG. 4 Scatter plot generation with positive Correlation

\subsection{Fuzzy rule generation:}

Fuzzy set $\mathrm{A}$ on a universe of discourse $\mathrm{U}$ is characterized by a membership function that takes values in the interval $[0,1]$.

In 1975 Professor Ebrahim Mamdani of London University built one of the first fuzzy systems to control a steam engine and boiler combination. He applied a set of fuzzy rules supplied by experience dhuman operators.

For ex

O Input x: research_funding

O Input y: project_staffing

O Output z: risk

\section{- Rules}

Rule1: If research_funding is adequate or project_staffing is small Then risk is low.

Rule2: If research_funding is marginal and project_staffing is large Then risk is normal.

Rule3: If research_funding is inadequate Then risk Is high.

\subsection{Map construction}

BSMs are of fundamental importance towards the understanding of the structure of a business. BSMs indicate which business parameters are related and which are particularly important.

In a Business Structure Map the following components are present

2.3.1Nodes- These are the business parameters located along the map's diagonal and represented by red squares or discs. The node represent the concept or entity.

Examples: profit, loss, expenses, customer satisfaction, hr satisfaction etc can be represented as node.

There are two type of nodes that is input and output. Input nodes are those which brings input to the firm and output goes are output nodes.[13]

Example: Revenue is input node and taxes are output .Input and output nodes are represented by 2 different colours. 
2.3.2Links - These are either strong or weak and are represented by black and grey connectors respectively. These links represent the strong and weak dependency between various parameters .these dependencies can be represented by the different colour connectors.

2.3.3Hubs - The red discs correspond to those parameters which are related to the highest number of other parameters.

Online self-rating system delivers the so called Business Structure Map which reflects the relationships between the various parameters of your business. The full power of our technology lies in its ability to analyze the complexity of systems of systems. Think of portfolios of financial products, companies or conglomerates of corporations and banks. How are they related to each other, How do they interact, Which are the most important ones, Which would cause the largest amount of damage in the event of default ,How would contagion propagate, All these and other questions may be answered with our tools in a truly innovative manner.

\subsection{Hubs and inactive nodes identification:}

The number of links in a node decide which node is hub and which node is inactive node. The node with the most relationships is called the hub while node with no links are inactive. Both kinds of nodes are clearly represented in the map. The hubs that have close relationships with other nodes appear as circular shapes while the inactive nodes are shown as white squares.

\section{Module 3: Analysis}

Static Analysis: The static analysis uses all the historical data to construct a Knowledge Map to give complete picture of companies.

Time Domain Analysis: In time domain analysis the data will be split into several continues periods or windows. For ex ,to view the salary of particular month or year.[14]

\section{VI.Results}

Knowledge map is useful for representing knowledge and for monitoring the health of companies. Furthermore sudden changes of the key features of the K.M should be taken seriously by policymakers as an alarm of a crisis. The main objective is to get good results from historical data so that the prediction and formation of policies. This method can be used in many fields, such as product design, management, medicine and air traffic control. maps mined from historical data are more valid and lose less information than those relying on the perceptions of experts..The Knowledge .Map mining method proposed by Marczyk eliminates the long iterative procedure and constructs a Knowledge .Map directly by analyzing the data resource. [14]

Based on knowledge map you can judge which parameters has the greatest impact on the company policy. You can see the relationship between employee salary and productivity.

(1) Identification of cause-effect relationships between variables.

(2) Visualization of how information flows within a given system.

(3) Ranking of variable importance indicated by hubs and inactive nodes.

When we will construct knowledge map with regular parameter, we will add few more parameters based on the output of Knowledge Map and which factors are affecting lot.

\section{VII.Conclusion}

This paper describes a mining method to construct knowledge maps utilizing historical data without the intervention of domain experts. The software is used to apply the method to analyze component stock corporations. The static analysis results show that the Knowledge Map is capable of discovering the structure of the examined systems given through fuzzy rules. Time domain analysis reveals the evolution of the main feature so the knowledge maps which can be used by policymakers to monitor the company health. The results demonstrate that the mining of knowledge main properties of the Knowledge Map can effectively indicate crises, which is not possible by conventional risk rating methods.

This idea is very much useful in automobile and aerospace industries as a design simulation. It will be also very helpful to other related areas such as textile industries, bank ,sales etc. Banking, E-commerce, HR, and Production industries.[15] 


\section{Acknowledgement}

The concepts presented represent the work of Dr.Jacek Marczyk. which is greatly acknowledge. The information in this document reflects solely the views of the authors.

\section{References}

[1]. B. Kosko, Fuzzy cognitive maps, International Journal of Man- Machine Studies, vol. 24, no. 3, pp.65-75,1986.

[2]. J. Aguilar, A survey about fuzzy cognitive maps papers, International Journal of Computational Cognition, vol. 3, no. 2, pp. 27$33,2005$.

[3]. L. Rodriguez-Repiso, R. Setchi, and J. L. Salmeron,Modelling IT projects success with fuzzy cognitive maps, Expert Systems with Applications, vol. 32, no. 2, pp. 543-559, 2007.

[4]. Z. Peng, B. Yang, C. Liu , Z. Tang, and J. Yang, Research on one fuzzy cognitive map classifier, (in Chinese), Application Research of Computers, vol. 26 , no. 5 , pp.1757-1759, 2009.

[5]. T. Hong and I. Han , Knowledge-based data mining of news information on the Internet using cognitive maps and neural networks, Expert Systems with Applications, vol. 23, no. 1, pp. 1-8, 2002

[6]. E. I. Papageorgiou , Learning algorithms for Fuzzy cognitive mapsła review study, IEEE Trans on Systems, Man and Cybernetics, vol. 42, no. 2, pp. 150-163, 2012.

[7]. J. A. Dickerson and B. Kosko, Virtual worlds as fuzzy cognitive maps Presence, vol. 3, no. 2, pp. 173-189, 1994

[8]. M. Schneider, E. Shnaider, A. Kande 1, and G. Chew , Constructing fuzzy cognitive maps, in Proc. 1995 IEEE International Conference on uzzy Systems, Yokohama, Japan, 1995, pp. 2281-2288.

[9]. K. E. Parsopoulos, E. I. Papageorgiou , P. P. Groumpos, and M. N. Vrahatis, A first study of Fuzzy cognitive maps learning using Particle swarm optimization, in Proc. 2003 Congress on Evolutionary Computation, 2003, pp. 14401447.

[10]. W. Stach, L. Kurgan , W. Pedrycz, and M. Reformat, Learning fuzzy cognitive maps with required precision using genetic algorithm approach, Electronics Letters, vol.40, no. 24, pp.1519-1520, 2004.

[11]. G. Allen and J. Marczyk, Tutorial on Complexity management for decision-making, pdf, 2012

[12]. J. Marczyk, A New Theory of Risk And Rating, Trento:EditriceUni Service, 2009.

[13]. D. V. Steward, The design structure matrix: A Method formanaging the design of complex systems, IEEE Transactions on Engineering Management, vol. EM-28, no. 3, pp.71-74, 1981.

[14]. S. Aumonier, Generalized correlation power Analysis , in Proc. ECRYPT Workshop on Tools For Cryptanalysis Krakw, Poland, 2007.

[15]. C. E. Shannon, A mathematical theory of communication, Bell System Technical Journal, vol. 27, pp.379-423, 1948.

[16]. B. Lent, A. Swami, and J. Widom, Clustering Associationrules, in Proc. 13th International Conference of DataEngineering,Birmingham, England, 1997, pp. 220. 